\title{
DETERMINAÇÃO POR SENSORIAMENTO REMOTO DA PRODUTIVIDADE PRIMÁRIA BRUTA DO PERÍMETRO IRRIGADO SÃO GONÇALO - PB
}

\author{
BERNARDO BARBOSA DA SILVA ${ }^{1}$, JOSICLÊDA DOMICIANO GALVÍNCIO ${ }^{1}$, SUZANA MARIA \\ GICO LIMA MONTENEGRO ${ }^{1}$, CÉLIA CRISTINA CLEMENTE MACHADO ${ }^{1}$, LEIDJANE MARIA \\ MACIEL DE OLIVEIRA ${ }^{1}$ E MAGNA SOELMA BESERRA DE MOURA $^{2}$
}

\author{
${ }^{1}$ Universidade Federal de Pernambuco (UFPE), Recife, PE, Brasil \\ ${ }^{2}$ Empresa Brasileira de Pesquisa Agropecuária (EMBRAPA), Petrolina, PE, Brasil
}

bbdasilva.ufpe@gmail.com, josicleda@hotmail.com, suzanam.ufpe@gmail.com, celiamachado.machado@ gmail.com, leidjaneoliveira@hotmail.com,magna@cpatsa.embrapa.br

Recebido Fevereiro de 2012 - Aceito Julho de 2012

\section{RESUMO}

O conhecimento da produtividade primária bruta - GPP (do inglês Gross Primary Production) é de fundamental importância nos estudos de mudanças climáticas, por estar diretamente relacionada ao carbono efetivamente extraído da atmosfera pelos diferentes ecossistemas terrestres. O presente trabalho objetivou determinar a GPP no perímetro irrigado São Gonçalo (PB), localizado no Semiárido brasileiro, com imagens TM - Landsat 5 e dados complementares de superfície. Foram adquiridas imagens TM - Landsat 5, sem presença de nuvens no ano de 2008, para obtenção da GPP diária em cinco dias selecionados. O modelo utilizado expressa a GPP em função da radiação fotossinteticamente ativa absorvida e da eficiência de uso da luz pela vegetação. Os resultados obtidos foram comparados aos valores da GPP extraídos do produto MOD17A2. As diferenças entre os valores da GPP obtida segundo as duas técnicas resultaram em Desvio Absoluto Médio - DAM de 0,91 $\mathrm{g} \mathrm{C} \mathrm{m}^{-2} \mathrm{dia}^{-1}$, Desvio Percentual Médio - DPM de 11,82\% e Raiz do Desvio Quadrático Médio - RDQM de 1,12 $\mathrm{g} \mathrm{C} \mathrm{m}^{-2} \mathrm{dia}^{-1}$. A integração da GPP em todo o Perímetro Irrigado São Gonçalo, durante os cinco dias selecionados, resultou na fixação de 200,3 a 295,3 ton C dia ${ }^{-1}$.

Palavras-chave: sequestro de carbono, saldo de radiação, Landsat 5, IVDN, eficiência de uso da luz

\begin{abstract}
ASSESSMENT OF GROSS PRIMARY PRODUCTION - GPP IN THE IRRIGATED PERIMETER SÃO GONÇALO - PB USING REMOTE SENSING

The Gross Primary Production (GPP) assessment and monitoring by remote sensing is of crucial importance in the climate changes studies given its direct connection to the effectively extracted carbon from the atmosphere by numerous earth ecosystems. The present work aims to assess the GPP in a Brazilian semi-arid irrigated area, using TM - Landsat 5 images and complementary surface meteorological data. Cloudless TM images were acquired from the year 2008 and were used to obtain daily GPP for five selected days. This model expresses GPP as a function of the absorbed photosynthetic active radiation and the efficiency of light use by vegetation. A comparison is made between the obtained results with TM images and GPP from MOD17A2. The difference between GPP values obtained by the two techniques resulted in an Average Absolute Difference - AAD of $0.91 \mathrm{~g}$ $\mathrm{C} \mathrm{m}^{-2} \mathrm{dia}^{-1}$, an Average Percentage Difference - APD of 11.82\% and Root Square Mean Difference RSMD of $1.12 \mathrm{~g} \mathrm{C} \mathrm{m}^{-2} \mathrm{dia}^{-1}$. The integration of the GPP over the irrigated São Gonçalo areas, during the five selected days, resulted in a carbon fixation between 200.3 and 295.3 ton $\mathrm{C}_{\text {day }}{ }^{-1}$.

Keywords: sequester of carbon, net radiation, Landsat 5, NDVI, light use efficiency
\end{abstract}




\section{INTRODUÇÃO}

Após a divulgação do IV Relatório do IPCC (IPCC, 2007), em fevereiro de 2007, o tema mudanças climáticas tem recebido grande destaque pela mídia e institutos científicos. Os gases do efeito estufa - GEE resultantes de atividades humanas têm sido apontados como os principais responsáveis pelas mudanças climáticas em curso no planeta. Embora não haja unanimidade no meio científico da responsabilidade das emissões antrópicas, vários centros de pesquisa internacionais têm recorrido a diversos líderes mundiais e chefes de estado para que sejam desprendidas ações que objetivamente promovam diminuição das emissões dos GEE e, ao mesmo tempo, para que se fomente o desenvolvimento de tecnologias de extração e armazenamento do carbono atmosférico.

Existem diversos métodos destinados à quantificação do carbono estocado na vegetação (Gallon et al., 2006; Lefsky et al., 2005; Santos e Costa, 2003), dentre os quais se destaca a técnica da covariância de vórtices turbulentos, muito empregada para quantificar o fluxo de $\mathrm{CO}_{2}$ em diversos ecossistemas florestais (Falge et al., 2002; Baldocchi, 2003; Aguiar et al., 2006; Oliveira et al., 2006; Kirschbaum et al., 2007; Beer et al., 2010; Cabral et al., 2011). Esta técnica goza de grande prestígio junto à comunidade científica, mas seu custo e operacionalidade muitas vezes se apresentam restritivos para vários grupos de pesquisa nos países em desenvolvimento. Outra questão que restringe o uso de tal técnica é a sua representatividade, que cobre apenas alguns quilômetros quadrados, salvo nos casos de áreas extensas e homogêneas. Nesse sentido, os métodos que empregam o sensoriamento remoto (Wu et al., 2009; Zhao et al., 2005; Xiao et al., 2004; Sims et al., 2008; Gitelson et al., 2008; Hilker et al., 2008; Li et al., 2007; Turner et al., 2006; Kalfas et al., 2011) apresentam como grande vantagem a ampla cobertura espacial, baixo custo e maior operacionalidade. Muitas dessas técnicas se fundamentam no conceito de eficiência de uso da luz - LUE (do inglês Light Use Efficiency) proposto por Monteith (1972). A sua aplicação prática resultou de grande contribuição dada por Field et al. (1995) e, mais recentemente, Bastiaanssen e Ali (2003), que empregaram o conceito de fração evaporativa com vistas a contabilizar o fator água no processo de fixação do carbono por parte da vegetação.

Diversos sensores orbitais têm sido empregados na determinação da GPP, destacando-se dentre eles, o ETM+ do Landsat 7 (Gitelson et al., 2008), o Moderate Resolution Imaging Spectroradiometer - MODIS (Running et al., 2004; Sakamoto et al., 2011) e o Advanced Very High Resolution Radiometer-AVHRR (Kitamoto et al., 2007). Muitos modelos da GPP baseados em sensoriamento remoto se fundamentam na relação entre a eficiência de uso da luz $-\varepsilon$, a fração da radiação fotossinteticamente ativa absorvida - fPAR e a radiação fotossinteticamente ativa - RFA. Um dos maiores desafios no uso de tais modelos consiste em se obter " $\varepsilon$ " numa grande área, isto em virtude de sua dependência de fatores ambientais e da própria vegetação. Uma das soluções consiste em relacionar " $\varepsilon$ " em função do seu valor máximo - $\varepsilon_{\max }$, mais as contribuições ambientais sintetizadas pela temperatura do ar e o status de água na vegetação (Field et al., 1995; Bastiaanssen e Ali, 2003). Outro elemento chave na modelagem da GPP é a RFA, em virtude de seu papel na fotossíntese e dada sua dependência à dinâmica da atmosfera. Embora mapas da GPP integrantes do produto MOD17A2 estejam disponíveis para diversos ecossistemas terrestres, o mesmo possui resolução de $1 \mathrm{~km}$ e é gerado para períodos de oito dias; além disso, a RFA é estimada com base em dados de radiação solar global extraídos de dados de re-análises. Nesse sentido, esta pesquisa objetiva avaliar a capacidade de fixação de carbono do Projeto Irrigado São Gonçalo - PISG, o mais importante do semiárido do estado da Paraíba, com base em observações do sensor TM do satélite Landsat 5, complementadas com medições da radiação solar efetuadas no interior do PISG. Não há registro do papel desempenhado pelos cultivos irrigados no Semiárido brasileiro, no que concerne ao sequestro de carbono.

\section{DADOS E METODOLOGIA}

\subsection{A área de estudo}

A área de estudo compreende o Perímetro Irrigado São Gonçalo - PISG, formado principalmente por fruticultura irrigada, Açude de São Gonçalo, áreas de vegetação nativa e agricultura de sequeiro (Figura 1). O recorte selecionado para a pesquisa possui 17,2 mil ha, com destaque para o rio Piranhas. O clima da região de estudo é classificado como semiárido, tipologia DdA', segundo classificação de Thornthwaite (VarejãoSilva et al., 1985), com estação chuvosa concentrada no período de fevereiro a maio. As médias anuais de temperatura do ar, precipitação e evapotranspiração potencial - ETp são respectivamente iguais a $25,3^{\circ} \mathrm{C}, 995,5 \mathrm{~mm}$ e $1480 \mathrm{~mm}$. Os solos predominantes na área são Latossolos e Cambissolos e a vegetação natural é classificada como Contato CaatingaFloresta Estacional e Formações Pioneiras com Influência Fluviomarinha.

\subsection{Imagens de satélite}

Foram obtidas imagens geradas pelo Mapeador Temático - TM do satélite Landsat 5, órbita 216 e ponto 65, junto ao Instituto de Pesquisas Espaciais - INPE. Essas imagens são compostas de sete bandas espectrais com resolução espacial de $30 \mathrm{~m} \times 30 \mathrm{~m}$, exceto a banda 6 (banda termal), com resolução 




Figura 1 - Recorte da área de estudo com destaque para o Perímetro Irrigado São Gonçalo, cidade de Sousa, rios do Peixe e Piranhas e o Açude da São Gonçalo, em combinação RGB123 de imagem TM Landsat 5 de 01 de novembro de 2008

de $120 \mathrm{~m}$ x $120 \mathrm{~m}$. A passagem do Landsat 5 na área de estudo ocorreu aproximadamente às $09 \mathrm{~h} 30 \mathrm{~min}$ (tempo local) nos dias estudados ( 29 de agosto, 14 de setembro, 01 e 17 de novembro e 19 de dezembro de 2008). Dados empregados no cômputo das diferentes componentes dos balanços de radiação e energia estão representados na Tabela 1.

\subsection{Produtividade primária bruta - GPP}

A produtividade primária bruta - GPP corresponde à fotossíntese bruta (Hunt Jr. et al., 2004; Xiao et al., 2004). A sua determinação com sensoriamento remoto pode ser feita ao se combinar o modelo de estimativa da radiação fotossinteticamente ativa absorvida - RFAA pela vegetação, proposto por Monteith (1972), com o modelo de eficiência de uso da luz na fotossíntese de Field et al. (1995), adaptado por Bastiaanssen e Ali (2003), que sugerem o emprego da fração evaporativa - FE como elemento quantificador do status da água pela vegetação. Essa fração evaporativa é obtida por meio do Surface Energy Balance Algorithm for Land - SEBAL. A radiação fotossinteticamente ativa - RFA corresponde à fração da radiação solar, situada entre $400 \mathrm{~nm}$ e $700 \mathrm{~nm}$, e na ausência de medições da mesma pode-se estimá-la em função da radiação solar global diária - Rs,24h. Destaque-se que apenas uma fração da RFA é absorvida pelo dossel vegetativo no processo de fixação do carbono. Na sequência, são apresentadas as equações empregadas no cômputo da GPP, que se fundamenta no modelo proposto por Monteith (1972), qual seja:

$$
\mathrm{GPP}=\mathrm{RFAA} \times \varepsilon=\mathrm{RFA} \times \mathrm{FPAR} \times \varepsilon
$$

em que, RFAA é a radiação fotossinteticamente ativa absorvida RFAA $\left(\mathrm{W} \mathrm{m}^{-2}\right)$ pela vegetação, $\varepsilon\left(\mathrm{g} \mathrm{C} \mathrm{MJ}^{-1}\right)$ é a eficiência do uso de luz da vegetação e FPAR representa a fração da radiação RFA, que é absorvida pela vegetação, a qual pode ser estimada em função da radiação solar global diária (Bastiaanssen e Ali, 2003):

$$
\mathrm{RFA}=0,48 \times \mathrm{Rs}, 24 \mathrm{~h}\left(\mathrm{~W} \mathrm{~m}^{-2}\right)
$$

Por sua vez, a fração da radiação RFA, que é absorvida pela vegetação, pode ser expressa em função do Índice de Vegetação da Diferença Normalizada - IVDN, proposta por Daughtry et al. (1995) e tem sido usada em outros estudos (Running et al., 2004; Bastiaanssen e Ali, 2003):

$$
\mathrm{RFAA}=\mathrm{RFA} \times(-0,161+1,257 \mathrm{IVDN})\left(\mathrm{Wm}^{-2} \mathrm{dia}^{-1}\right)
$$

A eficiência do uso de luz - $\varepsilon\left(\mathrm{g} \mathrm{C} \mathrm{MJ}^{-1}\right)$, embora dependa de outros elementos da comunidade vegetal, pode ser quantificada pelo modelo de Field et al. (1995), modificado por Bastiaanssen e Ali (2003):

$$
\varepsilon=\varepsilon^{\prime} \cdot \mathrm{T}_{1} \cdot \mathrm{T}_{2} \cdot \mathrm{FE}
$$

em que, $\varepsilon$ ' representa a eficiência máxima de uso da luz pela vegetação (considerada na pesquisa igual a $2,15 \mathrm{~g} \mathrm{C} \mathrm{MJ}^{-1}$ ), FE é a fração evaporativa, que reflete a contribuição do fator umidade na fotossíntese, e $\mathrm{T}_{1}$ e $\mathrm{T}_{2}$ refletem a contribuição da

Tabela 1 - Datas das imagens do TM, ângulo zenital do Sol - Z (grau), quadrado da razão entre a distância Terra-Sol e seu valor médio - dr, cosseno de Z - cos Z, temperatura do ar - Ta $\left({ }^{\circ} \mathrm{C}\right)$, umidade relativa do ar - UR (\%), pressão atmosférica - po (KPa), radiação fotossinteticamente ativa - RFA $\left(\mathrm{W} \mathrm{m}^{-2}\right)$ e transmitância atmosférica - $\tau$ oc,ins no momento da passagem do satélite na área de estudo em diferentes dias de 2008

\begin{tabular}{ccccccccc}
\hline \multicolumn{1}{c}{ Data } & $\mathrm{Z}$ (grau) & $\mathrm{d}_{\mathrm{r}}$ & $\cos \mathrm{Z}$ & $\mathrm{Ta}\left({ }^{\circ} \mathrm{C}\right)$ & $\mathrm{UR}(\%)$ & $\mathrm{p}_{\mathrm{o}}(\mathrm{KPa})$ & $\mathrm{RFA}\left(\mathrm{Wm}^{-2}\right)$ & $\tau_{\text {oc,ins }}$ \\
\hline 29/agosto & 35,30 & 0,9828 & 0,8161 & 29,1 & 46,2 & 98,1 & 387,8 & 0,737 \\
& & & & & & & & \\
14/setembro & 31,84 & 0,9912 & 0,8495 & 29,2 & 47,1 & 98,9 & 408,5 & 0,740 \\
11/novembro & 27,94 & 1,0174 & 0,8834 & 29,5 & 45,0 & 98,7 & 438,7 & 0,744 \\
17/novembro & 29,48 & 1,0244 & 0,8705 & 28,6 & 46,3 & 98,3 & 463,3 & 0,745 \\
19/dezembro & 33,53 & 1,0324 & 0,8336 & 29,6 & 47,5 & 93,4 & 416,2 & 0,737 \\
\hline
\end{tabular}


temperatura na eficiência de uso da luz pelas plantas, podendo ser calculados por:

$$
\begin{aligned}
& \mathrm{T}_{1}=0,8+0,02 \mathrm{~T}_{\mathrm{opt}}-0,0005 \mathrm{~T}_{\mathrm{opt}}^{2} \\
& \mathrm{~T}_{2}=\frac{1}{1+\exp \left(0,2 \mathrm{~T}_{\mathrm{opt}}-10-\mathrm{T}_{\text {dia }}\right)} \cdot \frac{1}{1+\exp \left[0,3\left(-\mathrm{T}_{\mathrm{opt}}-10+\mathrm{T}_{\text {dia }}\right)\right]}
\end{aligned}
$$

em que, $\mathrm{T}_{\mathrm{opt}}$ é a temperatura média do ar $\left({ }^{\circ} \mathrm{C}\right)$ do mês de máximo IVDN e $\mathrm{T}_{\text {dia }}$ é a temperatura média diária do ar $\left({ }^{\circ} \mathrm{C}\right)$. A FE representa a contribuição do elemento água na fotossíntese e pode ser determinada com base no balanço de energia segundo o SEBAL (Bastiaanssen et al., 1998; Silva e Bezerra, 2006; Allen et al., 2007; Bezerra et al., 2008, 2010). No SEBAL se obtém a densidade do fluxo de calor latente - LE $\left(\mathrm{W} \mathrm{m}^{-2}\right)$ como resíduo do balanço de energia à superfície, qual seja:

$$
\mathrm{LE}=\mathrm{Rn}-\mathrm{G}-\mathrm{H}
$$

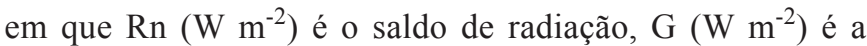
densidade do fluxo de calor no solo e $\mathrm{H}\left(\mathrm{W} \mathrm{m}^{-2}\right)$ é a densidade do fluxo de calor sensível. Os valores da fração evaporativa FE foram obtidos com base nos valores instantâneos de Rn, G e LE, segundo a expressão:

$$
F E=\frac{L E}{(R n-G)}
$$

Detalhes dos balanços de radiação e energia podem ser obtidos em Allen et al. (2007), Bezerra et al. (2010) e Silva et al. (2011). Com base nos dados diários da radiação solar global diária ( $\mathrm{MJ} \mathrm{m}^{-2}$ dia $\left.^{-1}\right)$, obtidos na estação meteorológica automática do INMET localizada em São Gonçalo, obteve-se a Radiação Fotossinteticamente Ativa ( $\mathrm{MJ} \mathrm{m}^{-2} \mathrm{dia}^{-1}$ ) para todos os dias selecionados para a pesquisa (Tabela 2).

\section{RESULTADOS}

Os dados complementares às imagens do TM - Landsat 5 empregados no cômputo da GPP estão representados na Tabela 2. Observa-se que dentre os dias com baixa presença de nebulosidade e que foram selecionados para o estudo, a temperatura do ar manteve-se entre 21,8 e $36,1^{\circ} \mathrm{C}$, enquanto que a umidade relativa do ar variou de 27,2 a $92,1 \%$. A radiação fotossinteticamente ativa - RFA manteve-se entre 12,13 e $13,85 \mathrm{MJ} \mathrm{m}^{-2} \mathrm{dia}^{-1}$. Uma variável importante no processo de cômputo da GPP é o IVDN, que apresentou valor médio em todos os dias selecionados próximo a 0,68 e por essa razão está sendo apresentado apenas o seu mapa temático do dia 01 de novembro de 2008 (Figura 2). Os parâmetros $\mathrm{T}_{1}$ e $\mathrm{T}_{2}$, que refletem a contribuição da temperatura na eficiência de uso da luz pelas plantas, situaram-se entre 0,945 e 0,970 , este último estando relacionado ao mês de novembro.

\subsection{Carbono fixado pela vegetação no PISG}

Na Figura 2 estão representados os mapas temáticos do IVDN (adimensional), saldo de radiação instantâneo (W $\mathrm{m}^{-2}$ ), fração evaporativa (adimensional) e produção primária bruta - GPP ( $\left.\mathrm{g} \mathrm{C} \mathrm{m}^{-2} \mathrm{dia}^{-1}\right)$ para o dia 01 de novembro de 2008. De acordo com a referida figura os valores do IVDN no PISG situaram-se acima de 0,6 , que os diferencia em relação às áreas não irrigadas. $\mathrm{O}$ mapa da fração evaporativa - FE também possibilita identificar padrões que definem claramente os contornos do PISG, com valores de FE geralmente superiores a 0,74 . O saldo de radiação $-\mathrm{Rn}\left(\mathrm{W} \mathrm{m}^{-2}\right)$ no instante da passagem do Landsat 5 sobre a área de estudo, ainda segundo a Figura 2 , apresentou valores entre 400 e $875 \mathrm{~W} \mathrm{~m}^{-2}$, resultantes da combinação de importantes elementos da superfície, como o IVDN, albedo e temperatura da superfície (Silva et al., 2011), o que faz aumentar o saldo radiativo. Embora a radiação solar incidente seja a mesma na área recortada (devido a ausência de nuvens), os valores de Rn são muito variáveis e registram os maiores valores no PISG. O mapa da GPP revela que em alguns pixels no PISG os valores da GPP superaram os $10 \mathrm{~g} \mathrm{C} \mathrm{m}^{-2} \mathrm{dia}^{-1}$, resultado da combinação da elevada radiação solar, alto índice de vegetação e elevada fração evaporativa.

Tabela 2 - Datas das imagens do TM, temperaturas do ar máxima - Tmax $\left({ }^{\circ} \mathrm{C}\right)$ e mínima- Tmin $\left({ }^{\circ} \mathrm{C}\right)$, umidades relativas máxima - Urmax $(\%)$ e mínima - Urmin (\%), radiação fotossinteticamente ativa diária - RFA (MJ m ${ }^{-2}$ dia $\left.^{-1}\right)$, valores de T1 e T2, referentes aos dias selecionados para a pesquisa em São Gonçalo - PB no ano de 2008.

\begin{tabular}{cccccccc}
\hline Data & Tmax & Tmin & URmax & URmin & RFA,24h & T1 & T2 \\
\hline 29/agosto & 32,4 & 22,6 & 73,0 & 57,1 & 12,23 & 0,970 & 0,967 \\
& & & & & & & \\
14/setembro & 32,2 & 22,0 & 77,1 & 64,9 & 12,13 & 0,970 & 0,953 \\
1/novembro & 32,5 & 24,0 & 87,3 & 66,0 & 13,15 & 0,970 & 0,945 \\
17/novembro & 33,1 & 25,4 & 92,1 & 76,3 & 13,19 & 0,970 & 0,945 \\
& & & & & & & \\
19/dezembro & 36,1 & 21,8 & 84,1 & 27,2 & 13,85 & 0,970 & 0,950
\end{tabular}




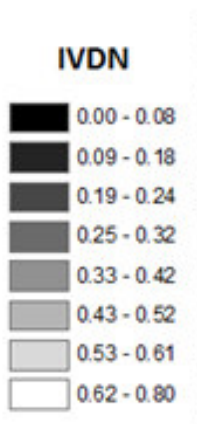

a)

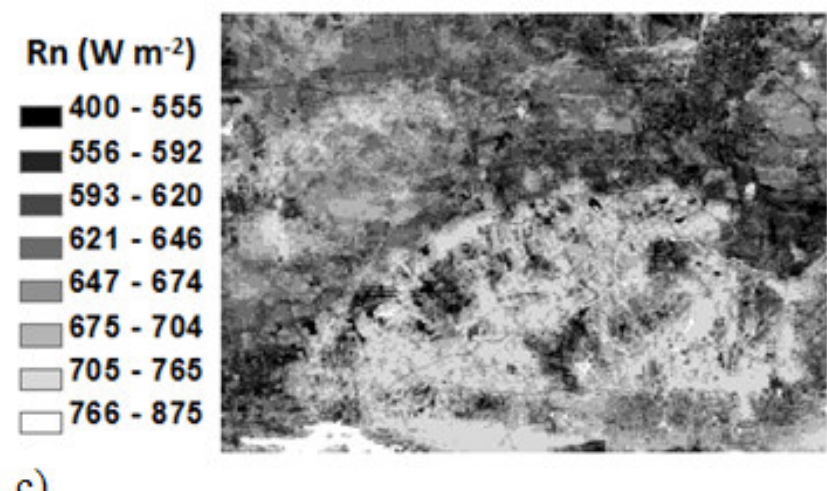

c)

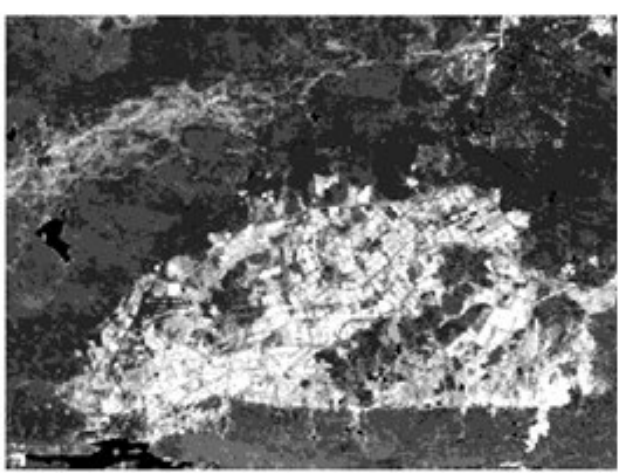

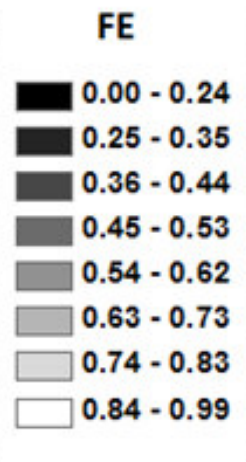

b)

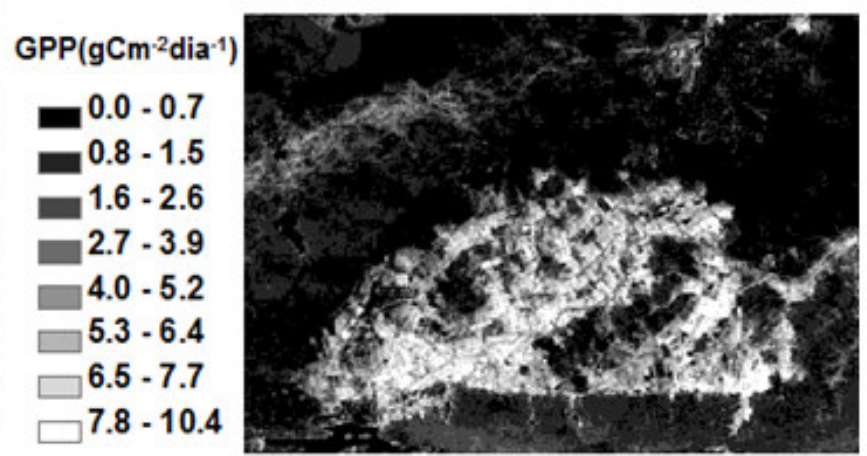

Figura 2 - Mapas temáticos: a) do Índice de Vegetação da Diferença Normalizada - IVDN; b) Fração Evaporativa - FE; c) Saldo de Radiação Instantâneo - Rn (W m $)$; e d) Produção Primária Bruta - GPP $\left(\mathrm{g} \mathrm{C} \mathrm{m}^{-2} \mathrm{dia}^{-1}\right)$, do dia 01 de novembro de 2008 no PISG.

Na Figura 3 são apresentados os mapas temáticos da GPP ( $\mathrm{g} \mathrm{C} \mathrm{m}^{-2} \mathrm{dia}^{-1}$ ) relativos aos dias 29 de agosto, 14 de setembro, 17 de novembro e 19 de dezembro de 2008. Observa-se que não há diferença perceptível entre os padrões da GPP, que revelam claramente que nas áreas do PISG os valores são substancialmente superiores àqueles das demais áreas do recorte selecionado, exceto nas proximidades do rio do Peixe, mas que também compreendem áreas irrigadas. De acordo com as legendas/escalas de cada um dos dias, os maiores valores da GPP foram registrados em dezembro, provavelmente resultado da maior oferta de RFA, que em 19 de dezembro foi igual a 13,85 $\mathrm{MJ} \mathrm{m}^{-2} \mathrm{dia}^{-1}$. Deve-se registrar que devido se tratar de áreas irrigadas, os mapas do IVDN no PISG não apresentaram grandes diferenças entre as imagens, daí as distinções na GPP estarem mais diretamente associadas às flutuações na radiação solar.

Para validação dos dados de GPP no PISG foram usados dados de GPP derivados do MODIS - Moderate Resolution Imaging Spectroradiometer, produto denominado de MOD17A2. Uma desvantagem deste produto em comparação ao obtido nesta pesquisa reside na sua resolução espacial, que é de 1 $\mathrm{km}$, e que o mesmo emprega dados de re-análise com dados em ponto de grade, o que pode provocar alguma incompatibilidade (Zhao et al., 2005). Para tanto, os dados de GPP obtidos com imagens TM foram re-amostrados para $1 \mathrm{~km}$ de modo que fosse possível a comparação com a GPP desta pesquisa com aquela do MOD17A2. Na Tabela 3 estão representados os dados da GPP de um pixel da área central do PISG, obtidos segundo as imagens $\mathrm{TM}-\mathrm{GPP}_{\mathrm{TM}}$ e segundo o produto MOD17A2 - GPP $\mathrm{MOD}_{\mathrm{M}}$. Os valores da $\mathrm{GPP}_{\mathrm{TM}}$ situaram-se entre 6,22 e 8,37 $\mathrm{g} \mathrm{C} \mathrm{m}^{-2} \mathrm{dia}^{-1}$, enquanto os da $\mathrm{GPP}_{\mathrm{MOD}}$ entre 5,57 e 8,46 $\mathrm{g} \mathrm{C} \mathrm{m}^{-2}$, com maior amplitude entre os dias selecionados. No estado americano de Nebraska, Kalfas et al. (2011) registraram valores da GPP em milho maiores que $20 \mathrm{~g} \mathrm{C} \mathrm{m}^{-2} \mathrm{dia}^{-1}$. Ao se comparar os valores obtidos nas duas bases, observou-se que o Desvio Absoluto Médio - DAM foi de $0,91 \mathrm{~g} \mathrm{C} \mathrm{m}^{-2} \mathrm{dia}^{-1}$, enquanto que o Desvio Relativo Médio - DRM e a Raiz do Desvio Quadrático Médio - RDQM foram respectivamente iguais a $11,81 \%$ e 1,12 g C $\mathrm{m}^{-2} \mathrm{dia}^{-1}$. Considerou-se ainda que os pixels das áreas irrigadas possuíssem IVDN $\geq 0,40$, o que resultou em 69.640 pixels no dia 29 de agosto e 57.066 em 19 de dezembro. A média dos valores da GPP no PISG situou-se entre 3,96 a 5,57 $\mathrm{g} \mathrm{C} \mathrm{m}^{-2}$ dia $^{-1}$, resultante da combinação da RFA, do fator temperatura e do fator água, que nas áreas do PISG não era restritivo. Computou-se também, o carbono total que fora fixado pela vegetação nas áreas com tal característica, qual seja, IVDN $\geq$ 0,40. Conforme se depreende da Tabela 3, o total de carbono 


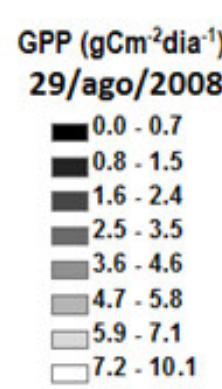

a)

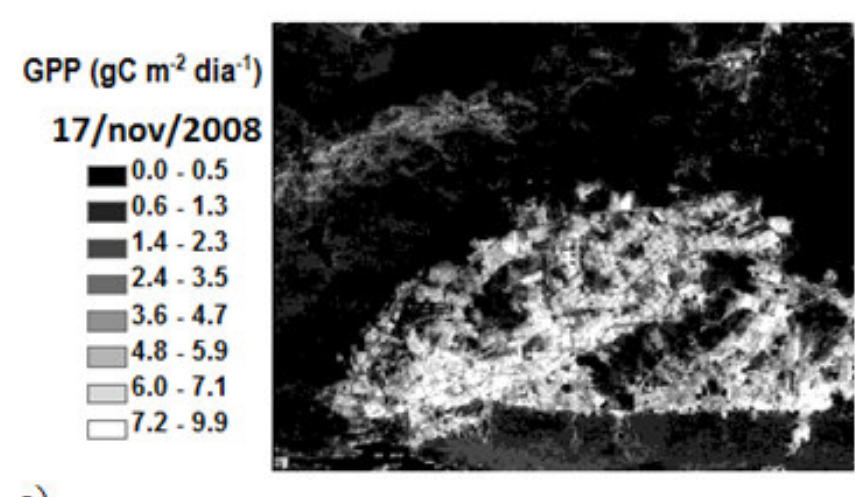

c)
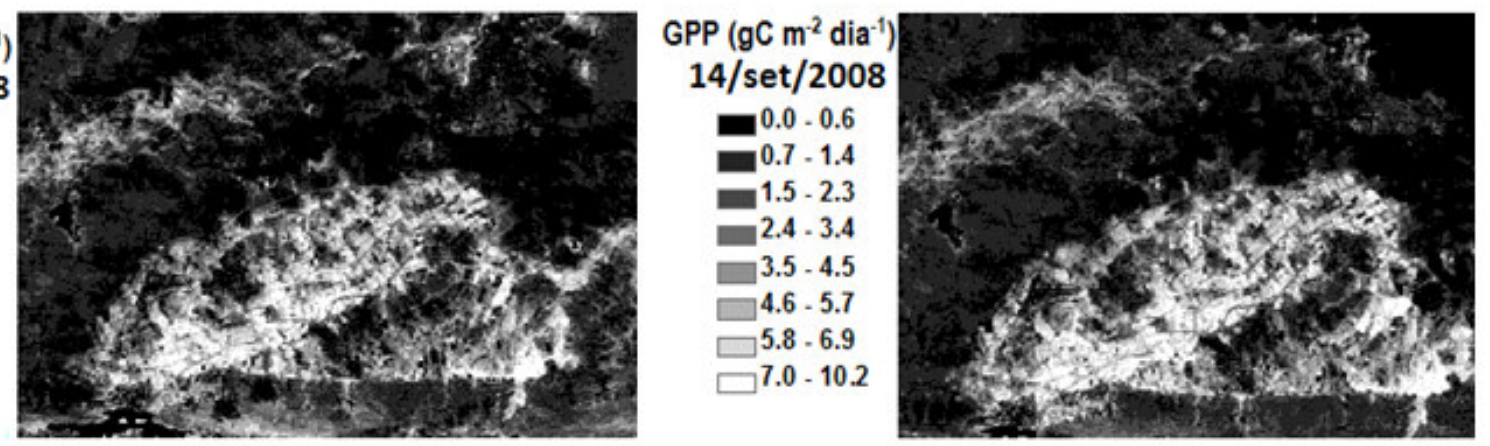

b)

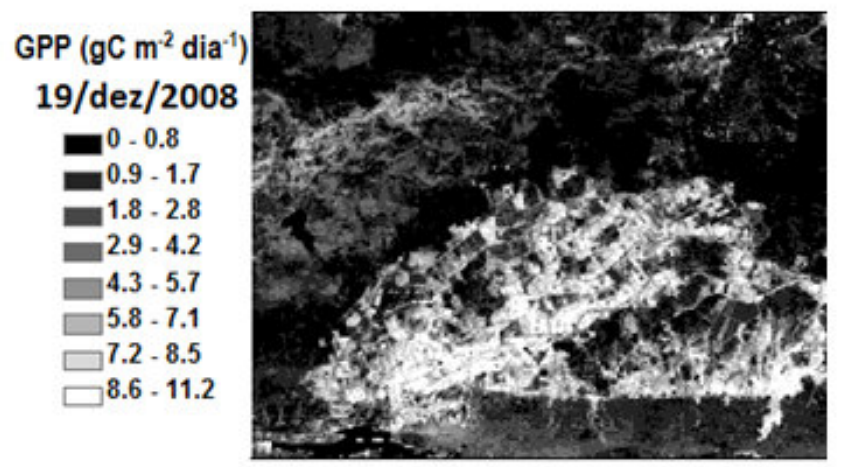

d)

Figura 3 - Mapas temáticos da Produção Primária Bruta - GPP (g C m${ }^{-2}$ dia $\left.^{-1}\right)$ dos dias: a) 29/agosto; b) 14/setembro; c) 17 de novembro; e d) 19/ dezembro do ano de 2008 no PISG.

Tabela 3 - Datas das imagens, GPP segundo imagens TM - GPPTM ( $\left.\mathrm{g} \mathrm{C} \mathrm{m}^{-2} \mathrm{dia}^{-1}\right)$, GPP segundo o produto MOD17 - GPPMOD $\left(\mathrm{g} \mathrm{C} \mathrm{m}^{-2}\right.$ $\left.\mathrm{dia}^{-1}\right)$, GPP médio no PISG - GPPmédio $\left(\mathrm{g} \mathrm{C} \mathrm{m}^{-2} \mathrm{dia}^{-1}\right)$, GPP integrado em todo o PISG - GPPPISG (ton $\mathrm{C} \mathrm{dia}^{-1}$ ) e GPP em área de sequeiro equivalente à do PISG - GPPSEQ (ton $\mathrm{C}$ dia $^{-1}$ ) para cada um dos dias selecionados para a pesquisa no ano de 2008.

\begin{tabular}{cccccc}
\hline Data & GPP $_{\mathrm{TM}}$ & $\mathrm{GPP}_{\mathrm{MOD} 17}$ & $\mathrm{GPP}_{\text {médio }}$ & $\mathrm{GPP}_{\mathrm{PISG}}$ & $\mathrm{GPP}_{\mathrm{SEQ}}$ \\
\hline 29/agosto & 7,02 & 8,46 & 3,96 & 248,2 & 21,6 \\
& & & & & \\
14/setembro & 6,31 & 8,21 & 4,32 & 212,4 & 16,7 \\
1/novembro & 7,06 & 6,87 & 5,05 & 233,1 & 15,3 \\
& & & & & \\
17/novembro & 6,22 & 5,57 & 4,74 & 200,3 & 14,0 \\
& & & & & \\
19/dezembro & 8,37 & 8,01 & 5,75 & 295,3 & 17,6 \\
\hline
\end{tabular}

fixado em cada um dos dias selecionados para a pesquisa no PISG variou de 200,3 a 295,3 ton $\mathrm{C} \mathrm{dia}{ }^{-1}$. Observou-se, com base na GPP de duas áreas representativas da área não irrigada e vegetação nativa com outra no interior da área irrigada, que na média a razão entre a irrigada e não irrigada foi de 14,3, o que indica que a GPP na área não irrigada, de mesmo tamanho das irrigadas, teria GPP total variando de 14,0 a 20,6 ton $\mathrm{C} \mathrm{dia}{ }^{-1}$, o que evidencia a importância da irrigação na fixação de carbono no Semiárido brasileiro. Deve-se registrar que os valores da GPP obtidos, em particular nas áreas não irrigadas e de vegetação, estão provavelmente superestimados, uma vez que a eficiência máxima de uso da luz não é ainda conhecida e presume-se que seja inferior ao considerado na pesquisa.

\section{CONCLUSÕES}

De acordo com os resultados obtidos, pode-se concluir que as diferenças entre a GPP obtida com imagens TM Landsat 5 e a derivada dos produtos MOD17A2 foram baixas $(11,7 \%)$, de modo que possibilitaram analisar detalhadamente as distinções entre áreas irrigadas e não irrigadas. O PISG apresenta um grande potencial de sequestro de carbono, uma vez que a 
diferença entre a GPP obtida nas áreas irrigadas, com aquelas com vegetação nativa não irrigada foi muito elevada $(14,3: 1)$.

\section{AGRADECIMENTOS}

Os autores agradecem ao Conselho Nacional de Desenvolvimento Científico e Tecnológico (CNPq) pelo apoio financeiro a esta pesquisa (Proc. 562512/2010-1) e por bolsa de Produtividade e Pesquisa e de doutorado concedida aos seus autores. Gostaríamos, ainda, de registrar nossa gratidão aos revisores e editores da RBMET, pelo laborioso trabalho e contribuições valiosas apresentadas.

\section{REFERÊNCIAS BIBLIOGRÁFICAS}

AGUIAR, R. G.; RANDOW, C. V.; PRIANTE FILHO, N.; MANZI, A. O.; AGUIAR, L. J. G.; CARDOSO, F. L. Fluxos de massa e energia em uma floresta tropical no sudoeste da Amazônia. Revista Brasileira de Meteorologia, v.21, n.3b, p.248-257, 2006.

ALLEN, R.; TASUMI, M.; TREZZA, R. Satellite-based energy balance for mapping evapotranspiration with internalized calibration (METRIC) - Model. Journal of Irrigation and Drainage Engineering, v.133, n.4, p.380-394, 2007.

BALDOCCHI, D. Assessing the eddy covariance technique for evaluating carbon dioxide exchange rates of ecosystems: past, present and future. Global Change Biology, v.9, p.479-492, 2003.

BASTIAANSSEN, W. G. M.; ALI, S. A new crop yield forecasting model based on satellite measurements applied across the Indus Basin, Pakistan. Agriculture, Ecosystems and Environment, v. 94, p.321-340, 2003.

BASTIAANSSEN, W. G. M.; MENENTI, M.; FEDDES, R. A.; HOLSLAG, A. A. M. A Remote Sensing Surface Energy Balance Algorithm for Land (SEBAL) - Formulation. Journal of Hydrology, v. 212-213, p.198-212, 1998.

BEER, C.; REICHSTEIM, M.; TOMELLERI, E. et al. Terrestrial gross carbon dioxide uptake: global distribution and covariation with climate. Science, v.329, 834-838, 2010.

BEZERRA, B. G.; SILVA, B. B. da; CORTEZ, J. R. G.; BRANDÃO, Z. N. Evapotranspiração real obtida através da relação entre o coeficiente dual de cultura da FAO-56 e o NDVI. Revista Brasileira de Meteorologia, v.25, p.404414, 2010.

BEZERRA, B. G.; SILVA, B. B. da; FERREIRA, N. J. Estimativa da evapotranspiração real diária utilizando-se imagens digitais TM - Landsat 5. Revista Brasileira de Meteorologia, v.23, n.3, p.305-317, 2008.

CABRAL, O. M. R.; GASH, J. H. C.; ROCHA, H. R.; MARSDEN, C.; LIGO, M. A. V.; FREITAS, H. C.;
TATSCH, J. D.; GOMES, E. Fluxes of $\mathrm{CO} 2$ above a plantation of Eucalyptus in southeast Brazil. Agricultural and Forest Meteorology, v.151, p.49-59, 2011.

DAUGHTRY C.S.T.; McMURTREY III, J. E.; CHAPPELLE, E. W.; DULANEY, W. P.; IRONS, J. R.; SATTERWHITE, M. B. Potential for discriminating crop residues from soil by reflectance and fluorescence. Agronomy Journal, v.87, n.2, p.165-171, 1995.

FALGE, E.; BALDOCCHI, D.; TENHUNEN, J.; AUBINET, M. et al. Seasonality of ecosystem respiration and gross primary production as derived from FLUXNET measurements. Agricultural and Forest Meteorology, v.113, n.1-4, p.5374. 2002.

FIELD, C. B.; RANDERSON, J. T.; MALMSTROM, C. M. Global net primary production: combining ecology and remote sensing. Remote Sensing of Environment, v.51, p.74-88, 1995.

GALLON, M. M. P.; SANCHES, L.; PAULO, S. R. DE. Fluxo e perfil de dióxido de carbono no dossel uma floresta tropical de transição amazônica. Revista Brasileira de Meteorologia, v.21, n.3, p.79-88, 2006.

GITELSON, A. A.; VIÑA, A.; MASEK, J. G.; VERMA, S. B.; SUYKER, A.E. Synoptic Monitoring of Gross Primary Productivity of Maize Using Landsat Data. IEEE Geosciences and Remote Sensing Letters, v.5, n.2, p.133-137, 2008.

HILKER, T.; COOPS, N. C.; WULDER, M. A.; BLACK, T. A.; GUY, R. D. The use of remote sensing in light use efficiency based models of gross primary production: A review of current status and future requirements. Science of the Total Environment, v.404, p.411- 423, 2008.

HUNT JR. E. R.; KELLY, R. D.; SMITH, W.K.; FAHNESTOCK. J. T.; WELKER, J. M.; REINERS, W. A. Estimation of Carbon Sequestration by Combining Remote Sensing and Net Ecosystem Exchange Data for Northern Mixed-Grass Prairie and Sagebrush-Steppe Ecosystems. Environmental Management, v.33, Supplement 1, p. 432-441, 2004.

IPCC - INTERGOVERNMENTAL PANEL ON CLIMATE CHANGE. Summary for Policy makers - Contribution of Work Group I to the Fourth Assessment Report of the Intergovernmental Panel on Climate Change. 2007.

KALFAS, J. L.; XIAO, X.; VANEGAS, D. X.; VERMA, S. B.; SUYKER, A. E. Modeling gross primary production of irrigated and rain-fed maize using MODIS imagery and $\mathrm{CO} 2$ flux tower data. Agricultural and Forest Meteorology, v.151, p.1514-1528, 2011.

KITAMOTO, T.; UEYAMA, M.; HARAZONO, Y.; IWATA, T.; YAMAMOTO, S. Applications of NOAA/AVHRR and observed fluxes to estimate 3 regional carbon fluxes over black spruce forests in Alaska. Journal of Agricultural Meteorology, v.63, p.171-183, 2007. 
KIRSCHBAUM, M. U. F.; KEITH, H.; LEUNING, R.; CLEUGH, H. A.; JACOBSEN, K. L.; VAN GORSEL, E.; RAISON, R. J. Modeling net ecosystem carbon and water exchange of a temperate Eucalyptus delegatensis forest using multiple constraints. Agricultural and Forest Meteorology, v.145, p.48-68, 2007.

LEFSKY, M. A.; TURNER, D. P.; GUZY, M.; COHEN, W. B. Combining lidar estimates of aboveground biomass and Landsat estimates of stand age for spatially extensive validation of modeled forest productivity. Remote Sensing of Environment, v.95, p.549-558, 2005.

LI, Z., YU, G., XIAO, X., LI, Y., ZHAO, X., REN, C., ZHANG, L., FU, Y. Modeling gross primary production of alpine ecosystems in the Tibetan Plateau using MODIS images and climate data. Remote Sensing of Environment, v.107, p.510-519, 2007.

MONTEITH, J. L. Solar radiation and productivity in tropical ecosystems. Journal of Applied Ecology, v.9, p.747-766, 1972.

OLIVEIRA, M. B. L.; SANTOS, A. J. B.; MANZI, A. O.; ALVAlÁ, R. C. S.; CORREIA, M. F.; MOURA, M. S. B. Trocas de energia e fluxo de carbono entre a vegetação de Caatinga e atmosfera no Nordeste brasileiro. Revista Brasileira de Meteorologia, v.21, n.3b, p. 378-386, 2006.

RUNNING, S. W.; NEMANI, R. R.; HEINSCH, F. A.; ZHAO, M. S.; REEVES, M.; HASHIMOTO, H. A continuous satellite-derived measure of global terrestrial primary production. Bioscience, v.54, n.6, p. 547-560, 2004.

SAKAMOTO, T.; GITELSON, A.; WARDLOW, B. D.; VERMA, S. B.; SUYKER, A. E. Estimating daily gross primary production of maize only on MODIS WDRVI and shortwave radiation data. Remote Sensing of Environment, v.115, p.3091-3101, 2011.

SANTOS, S. N. M.; COSTA, M. H. Simulações de fluxo de carbono em um ecossistema de floresta tropical. Revista Brasileira de Meteorologia, v.18, n.1, p.87-96, 2003.
SILVA, B. B. da; BEZERRA, M. V. C. Determinação dos fluxos de calor sensível e latente na superfície utilizando imagens TM - Landsat 5. Revista Brasileira de Agrometeorologia, v. 14, n. 2, p. 174-186, 2006.

SILVA, B. B. da; BRAGA, A. C.; BRAGA, C. C. Balanço de radiação no perímetro irrigado São Gonçalo - $\mathrm{PB}$ mediante imagens orbitais. Revista Caatinga, v.24, p.145-152, 2011. SIMS, D. A.; RAHMAN, A. F.; CORDOVA, V. D.; EL-MASRI, B. Z.; BALDOCCHI, D. D. et al. A new model of gross primary productivity for North American ecosystems based solely on the enhanced vegetation index and land surface temperature from MODIS. Remote Sensing of Environment, v.112, n.4, p.1633-1646, 2008.

TURNER, D. P.; RITTS, W. D.; ZHAO, M. S.; KURC, S. A.; DUNN, A. L.; WOFSY, S. C., et al. Assessing interannual variation in MODIS-based estimates of gross primary production. IEEE Transactions on Geosciences and Remote Sensing, v.44, p.1899-1907, 2006.

VAREJÃO-SILVA, M. A.; BRAGA, C. C.; SILVA, B. B. da. Atlas climatológico do Estado da Paraíba. Relatório Técnico, 59p, 1985.

WU, C.; NIU, Z.; TANG, Q.; HUANG, W.; RIVARD, B.; FENG, $\mathrm{J}$. Remote estimation of gross primary production in wheat using chlorophyll-related vegetation indices. Agricultural and Forest Meteorology, v.149, p.1015-1021, 2009.

XIAO, X. M.; ZHANG, Q. Y.; BRASWELL, B.; URBANSKI, S.; BOLES, S.; WOFSY, S.; MOORE, B.; OJIMA, D. Modeling gross primary production of temperate deciduous broadleaf forest using satellite images and climate data. Remote Sensing of Environment, v.91, n.2, p.256-270, 2004.

ZHAO, M.; HEINSCH, F. A.; NEMANI, R. R.; RUNNING, S. W. Improvements of the MODIS terrestrial gross and net primary production global data set. Remote Sensing of Environment, v.95, p.164-176, 2005. 